\title{
Searches for low mass dark bosons
}

\section{Andrea Mauri*t}

University of Zurich, Switzerland

E-mail: a.mauri@cern.ch

A search is presented for a hidden-sector boson, $\chi$, produced in the decay $B^{0} \rightarrow K^{*}(892)^{0} \chi$, with $K^{*}(892)^{0} \rightarrow K^{+} \pi^{-}$and $\chi \rightarrow \mu^{+} \mu^{-}$. The search is performed using a $p p$-collision data sample collected at $\sqrt{s}=7$ and $8 \mathrm{TeV}$ with the LHCb detector, corresponding to integrated luminosities of 1 and $2 \mathrm{fb}^{-1}$ respectively. No significant signal is observed in the mass range $214 \leq m_{\chi} \leq$ $4350 \mathrm{MeV}$, and upper limits are placed on the branching fraction product $\mathscr{B}\left(B^{0} \rightarrow K^{*}(892)^{0} \chi\right) \times$ $\mathscr{B}\left(\chi \rightarrow \mu^{+} \mu^{-}\right)$as a function of the mass and lifetime of the $\chi$ boson. These limits place the most stringent constraints to date on many theories that predict the existence of additional low-mass dark bosons.

The European Physical Society Conference on High Energy Physics

22-29 July 2015

Vienna, Austria

* Speaker.

${ }^{\dagger}$ On the behalf of the LHCb collaboration. 


\section{Introduction}

Most extensions of the Standard Model (SM) that address the problem of the existence of Dark Matter, postulate the existence of a hidden sector, see for example the review in Ref. [1]. Particles of the hidden sector are singlets with respect to the SM gauge number, however they can interact with SM particles via kinetic mixing. In this analysis a search for a light scalar particle (dark scalar boson, $\chi$ ) belonging to the secluded sector and mixing with Higgs boson is performed. Concrete examples of such models are theories where such a $\chi$ field was responsible for an inflationary period in the early universe [2], and the associated inflaton particle is expected to have a mass in the range $270<m(\chi)<1800 \mathrm{MeV}$. Another class of models invokes the axial-vector portal [3] in theories of dark matter that seek to address the cosmic-ray anomalies, and to explain the suppression of charge-parity (CP) violation in strong interactions [4]. These theories postulate an additional fundamental symmetry, the spontaneous breaking of which results in a particle called the axion [5]. The energy scale, $f(\chi)$, at which the symmetry is broken lies in the range $1 \lesssim f(\chi) \lesssim 3$ $\mathrm{TeV}[6]$.

\section{Search for $B^{0} \rightarrow K^{*}(892)^{0} \chi\left(\rightarrow \mu^{+} \mu^{-}\right)$}

The decay $B^{0} \rightarrow K^{* 0} \chi$, with $K^{* 0} \rightarrow K^{+} \pi^{-}$and $\chi \rightarrow \mu^{+} \mu^{-}$is studied to search for such a hidden-sector particle. An enhanced sensitivity to hidden-sector bosons arises because the $b \rightarrow s$ transition is mediated by a top quark loop at leading order (Fig.1). Therefore, a $\chi$ boson with $2 m(\mu)<m(\chi)<m\left(B^{0}\right)-m\left(K^{* 0}\right)$ and a sizable top quark coupling (obtained via mixing with the Higgs sector), could be produced at a substantial rate in such decays.

Similar searches have been performed in the past by B-factories [7, 8], they were the most stringent direct constraints on a light scalar dark boson. Their exclusion limits on the coupling (i.e. mixing angle) between the Higgs and the dark boson field lie between $7 \times 10^{-4}$ and $5 \times 10^{-3}$, whith the most sensitive region just below the $J / \psi$ threshold [9].

This search is performed with the full Run I dataset collected with the LHCb detector corresponding to an integrated luminosity of $3.0 \mathrm{fb}^{-1}$.

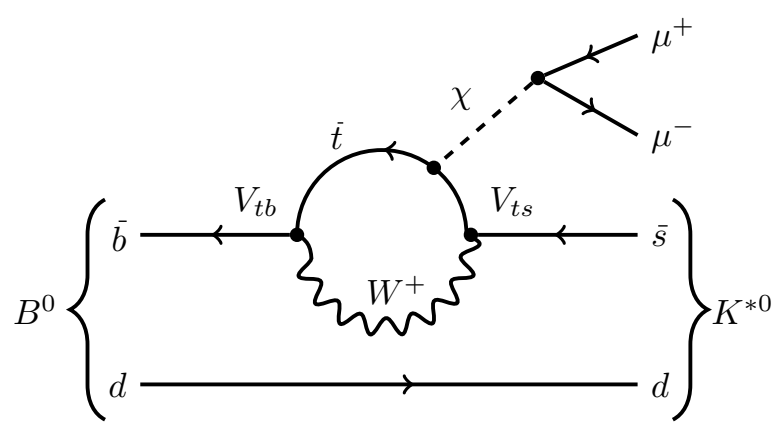

Figure 1: Feynman diagram for the decay $B^{0} \rightarrow K^{* 0} \chi$, with $\chi \rightarrow \mu^{+} \mu^{-}$. 


\section{Selection and strategy}

Depending on the strength of the mixing with the Higgs boson and its mass, the particle $\chi$ can decay in a secondary vertex, displaced from the $B^{0} \rightarrow K^{* 0} \chi$ decay vertex. In order to increase the sensitivity, two regions of reconstructed di-muon lifetime, $\tau\left(\mu^{+} \mu^{-}\right)$, are defined for each $m(\chi)$ considered in the search: a prompt region, $\left|\tau\left(\mu^{+} \mu^{-}\right)\right|<3 \sigma\left[\tau\left(\mu^{+} \mu^{-}\right)\right]$, and a displaced region, $\tau\left(\mu^{+} \mu^{-}\right)>3 \sigma\left[\tau\left(\mu^{+} \mu^{-}\right)\right]$, where $\sigma\left[\tau\left(\mu^{+} \mu^{-}\right)\right]$is the lifetime resolution. When setting a limit on the branching fraction the two regions are combined as a joint likelihood, $\mathscr{L}=\mathscr{L}^{\text {prompt }} \cdot \mathscr{L}^{\text {displaced }}$. These two regions correspond to the two possible scenarios: the former is sensitive to short lifetime dark boson, it is characterized by high reconstruction efficiency but it is highly contaminated by the irreducible SM background $B^{0} \rightarrow K^{* 0} \mu^{+} \mu^{-}$; the latter suffers of lower reconstruction efficieny but offers a very clear signature thanks to lower background yields.

A multivariate selection is applied to reduce the background, the uBoost algorithm [10] is employed to ensure that the performance is nearly independent of $m(\chi)$ and $\tau(\chi)$. The inputs to the algorithm include $B^{0}$ transverse momentum, various topological features of the decay, the muon identification quality, and isolation criteria. Only candidates with invariant mass $m_{B^{0}}$ within $50 \mathrm{MeV}$ of the known $B^{0}$ mass are selected. Then, the reconstructed $m_{B^{0}}$ is constrained to its known value to improve the resolution of the dimuon mass, that results to be less than $8 \mathrm{MeV}$ over the entire $m\left(\mu^{+} \mu^{-}\right)$range, and as small as $2 \mathrm{MeV}$ below $220 \mathrm{MeV}$.

The strategy described in Ref. [11] is adopted: the $m\left(\mu^{+} \mu^{-}\right)$distribution is scanned for an excess of $\chi$ signal candidates over the expected background. Since all the theoretical models predict the dark boson $\chi$ to have negligible width compared to the detector resolution, the signal window is entirely determinated by the di-muon mass resolution and is defined to be $\pm 2 \sigma\left[m\left(\mu^{+} \mu^{-}\right)\right]$around the tested mass. The step sizes in $m(\chi)$ are $\sigma\left[m\left(\mu^{+} \mu^{-}\right)\right] / 2$. In order to avoid experimenter bias, all aspects of the search are fixed without examining the $B^{0} \rightarrow K^{* 0} \chi$ candidates.

Narrow resonances are vetoed by excluding the regions near the $\omega, \phi, J / \psi, \psi(2 S)$ and $\psi(3770)$ resonances. These regions are removed in both the prompt and displaced samples.

\section{Results and exclusion limits}

Figure 2 shows the $m\left(\mu^{+} \mu^{-}\right)$distributions for the number of observed candidates in both the prompt and displaced regions. The observation is consistent with the background only hypothesis with a $p$-value of about $80 \%$, therefore an upper limit on $\mathscr{B}\left(B^{0} \rightarrow K^{* 0} \chi\left(\rightarrow \mu^{+} \mu^{-}\right)\right)$is set. Figure 3 shows the upper limits both on the absolute branching fraction $\mathscr{B}\left(B^{0} \rightarrow K^{* 0} \chi\left(\mu^{+} \mu^{-}\right)\right)$and on the relative ratio to the normalization channel $\mathscr{B}\left(B^{0} \rightarrow K^{* 0} \mu^{+} \mu^{-}\right)$in the $1.1<m^{2}\left(\mu^{+} \mu^{-}\right)<6.0$ $\mathrm{GeV}^{2}$ region. Limits are set at the $95 \%$ confidence level (CL) for several values of $\tau(\chi)$. The limits become less stringent for higher values of $\tau(\chi)$, as the probability of the $\chi$ boson decaying within the LHCb's silicon vertex detector decreases.

Figure 4 shows the interpretation of the exclusion limit in term of two benchmark models: the inflaton model of Ref. [12], which only considers $m(\chi)<1 \mathrm{GeV}$, and the axion model of Ref. [3]. In the first case, constraints are placed on the mixing angle between the Higgs and inflaton fields, $\theta$, which exclude most of the previously allowed region. For the latter, exclusion regions are set in the limit of large ratio of Higgs-doublet vacuum expectation values, $\tan \beta \gtrsim 3$, for charged-Higgs 


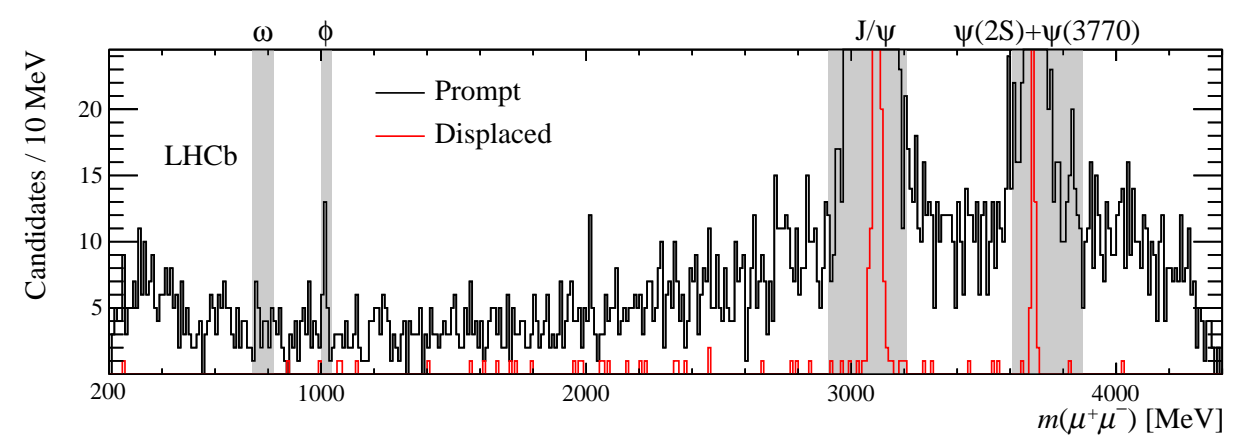

Figure 2: Distribution of $m\left(\mu^{+} \mu^{-}\right)$in the (black) prompt and (red) displaced regions. The shaded bands denote regions where no search is performed due to (possible) resonance contributions. The $J / \psi, \psi(2 S)$ and $\psi(3770)$ peaks are suppressed to better display the search region.

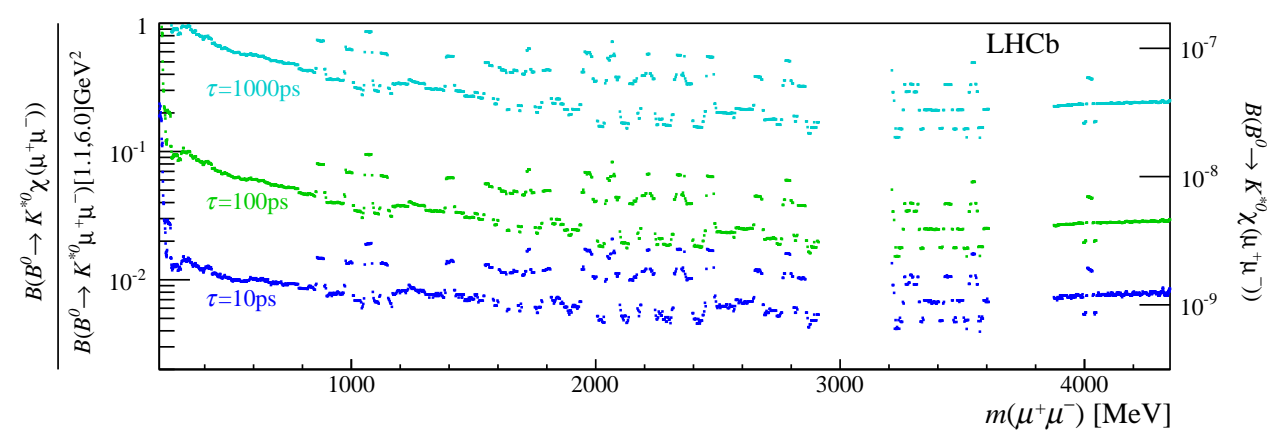

Figure 3: Upper limit on the (left-axis) ratio of branching fractions $\mathscr{B}\left(B^{0} \rightarrow K^{* 0} \chi\left(\mu^{+} \mu^{-}\right)\right) / \mathscr{B}\left(B^{0} \rightarrow\right.$ $\left.K^{* 0} \mu^{+} \mu^{-}\right)$, where the $B^{0} \rightarrow K^{* 0} \mu^{+} \mu^{-}$decay has $1.1<m^{2}\left(\mu^{+} \mu^{-}\right)<6.0 \mathrm{GeV}^{2}$ and (right-axis) on $\mathscr{B}\left(B^{0} \rightarrow\right.$ $\left.K^{* 0} \chi\left(\mu^{+} \mu^{-}\right)\right)$as a function of the dimuon mass. The limits are given at $95 \%$ confidence level. Limits are presented for three different lifetimes of the dark boson. The sparseness of the data leads to rapid fluctuations in the limits. The relative limits for $\tau<10$ ps are between $0.005-0.05$ except near $2 m(\mu)$.

masses $\mathrm{m}(h)=1$ and $10 \mathrm{TeV}$. The branching fraction of the axion into hadrons varies greatly in different models, the results for two extreme cases are shown: $\mathscr{B}(\chi \rightarrow$ hadrons $)=0$ and 0.99 .

\section{Conclusion}

In summary, a search is performed for light scalar dark boson in the decay $B^{0} \rightarrow K^{* 0} \chi(\rightarrow$ $\left.\mu^{+} \mu^{-}\right)$using $p p$-collision data collected at 7 and $8 \mathrm{TeV}$. No evidence of signal is observed, and upper limits are placed on $\mathscr{B}\left(B^{0} \rightarrow K^{* 0} \chi\right) \times \mathscr{B}\left(\chi \rightarrow \mu^{+} \mu^{-}\right)$. This is the most sensitive search to date over the entire accessible mass range and stringent constraints are placed on theories that predict the existence of additional scalar or axial-vector fields. 

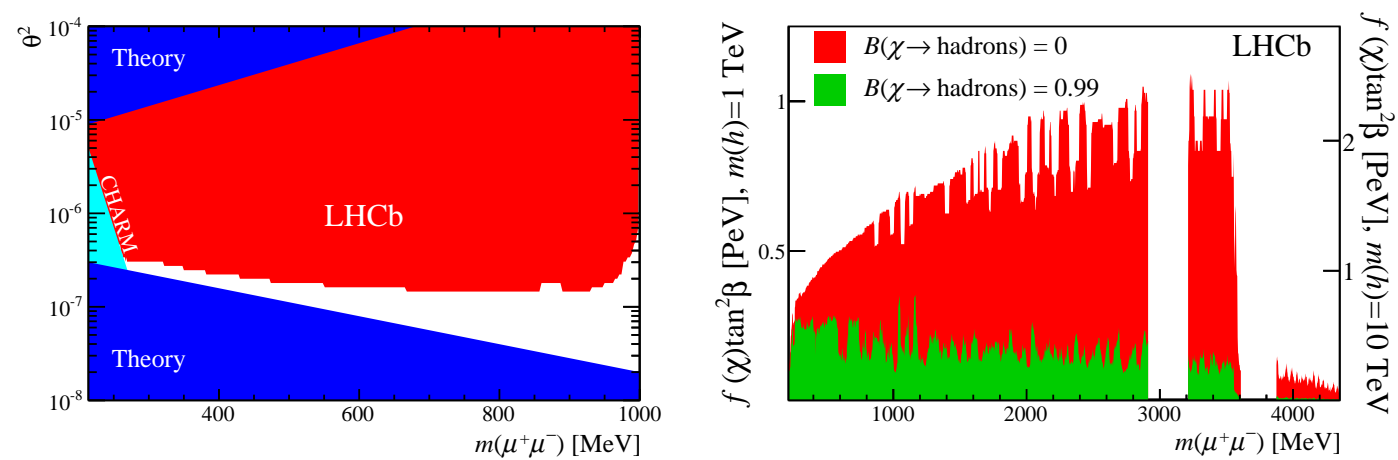

Figure 4: Exclusion regions at 95\% CL: (left) constraints on the inflaton model of Ref. [12]; (right) constraints on the axion model of Ref. [3]. The regions excluded by the theory [12] and by the CHARM experiment [13] are also shown.

\section{References}

[1] R. Essig et al., arXiv:1311.0029 [hep-ph].

[2] F. Bezrukov and D. Gorbunov, JHEP 1005, 010 (2010) [arXiv:0912.0390 [hep-ph]].

[3] M. Freytsis, Z. Ligeti and J. Thaler, Phys. Rev. D 81, 034001 (2010) [arXiv:0911.5355 [hep-ph]].

[4] R. D. Peccei, Lect. Notes Phys. 741, 3 (2008) [hep-ph/0607268].

[5] R. D. Peccei and H. R. Quinn, Phys. Rev. Lett. 38, (1977) 1440

[6] Y. Nomura and J. Thaler, Phys. Rev. D 79, 075008 (2009) [arXiv:0810.5397 [hep-ph]].

[7] J. P. Lees et al. [BaBar Collaboration], Phys. Rev. D 86, 032012 (2012) [arXiv:1204.3933 [hep-ex]].

[8] J.-T. Wei et al. [Belle Collaboration], Phys. Rev. Lett. 103, 171801 (2009) [arXiv:0904.0770 [hep-ex]].

[9] M. J. Dolan, F. Kahlhoefer, C. McCabe and K. Schmidt-Hoberg, JHEP 1503, 171 (2015) [JHEP 1507, 103 (2015)] [arXiv:1412.5174 [hep-ph]].

[10] J. Stevens and M. Williams, JINST 8, P12013 (2013) [arXiv:1305.7248 [nucl-ex]].

[11] M. Williams, JINST 10, no. 06, P06002 (2015) [arXiv:1503.04767 [hep-ex]].

[12] F. Bezrukov and D. Gorbunov, Phys. Lett. B 736, 494 (2014) [arXiv:1403.4638 [hep-ph]].

[13] F. Bergsma et al. [CHARM Collaboration], Phys. Lett. B 157, 458 (1985). 